\title{
Transitional Metal Catalytic Pyrite Cathode Enables Ultrastable Four-Electron-Based All-Solid-State Lithium Batteries
}

Hongli Wan ${ }^{a, b}$, Gaozhan Liu ${ }^{a, b}$, Yanle Li ${ }^{a, c}$, Wei Weng ${ }^{a, b}$, Jean Pierre Mwizerwa ${ }^{a, b}$, Ziqi Tian $a, c *$, Liang Chen ${ }^{a, b}$, Xiayin Yao ${ }^{a, b *}$

${ }^{a}$ Ningbo Institute of Materials Technology and Engineering, Chinese Academy of Sciences, Ningbo 315201, P. R. China

${ }^{\mathrm{b}}$ Center of Materials Science and Optoelectronics Engineering, University of Chinese Academy of Sciences, Beijing 100049 , P. R. China

${ }^{\mathrm{C} F u j i a n}$ Institute of Innovation, Chinese Academy of Sciences, Fuzhou, Fujian, 350002, P.R. China

*Corresponding authors: yaoxy@nimte.ac.cn (X.Y. Yao) and tianziqi@nimte.ac.cn (Z. Q. Tian) 


\section{Supporting Figures}

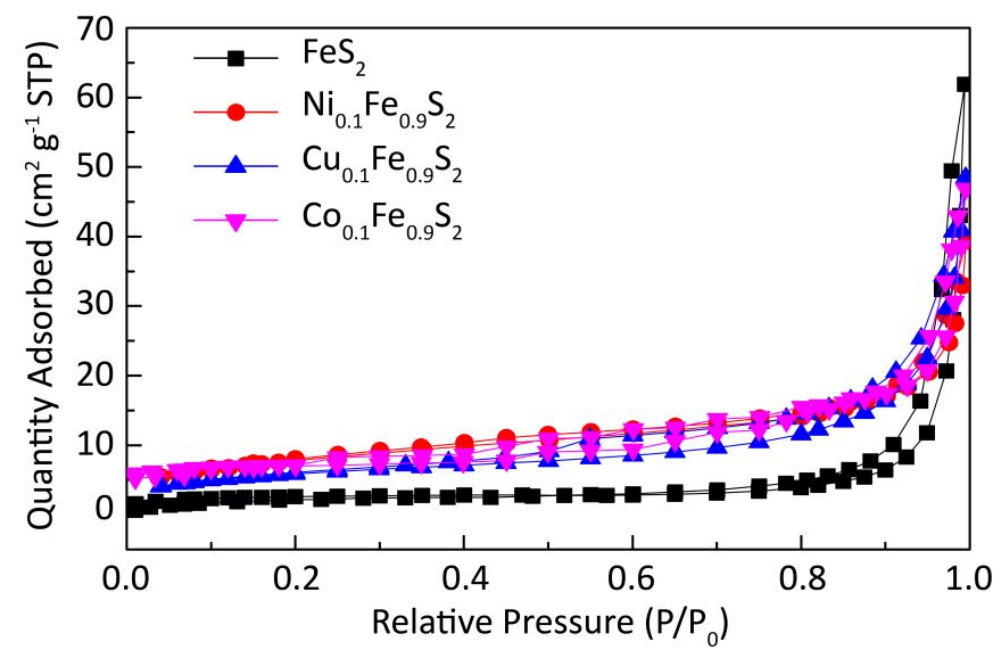

Figure S1. Nitrogen adsorption-desorption isotherm curves of $\mathrm{FeS}_{2}, \mathrm{Ni}_{0.1} \mathrm{Fe}_{0.9} \mathrm{~S}_{2}, \mathrm{Cu}_{0.1} \mathrm{Fe}_{0.9} \mathrm{~S}_{2}$ and $\mathrm{Co}_{0.1} \mathrm{Fe}_{0.9} \mathrm{~S}_{2}$. 


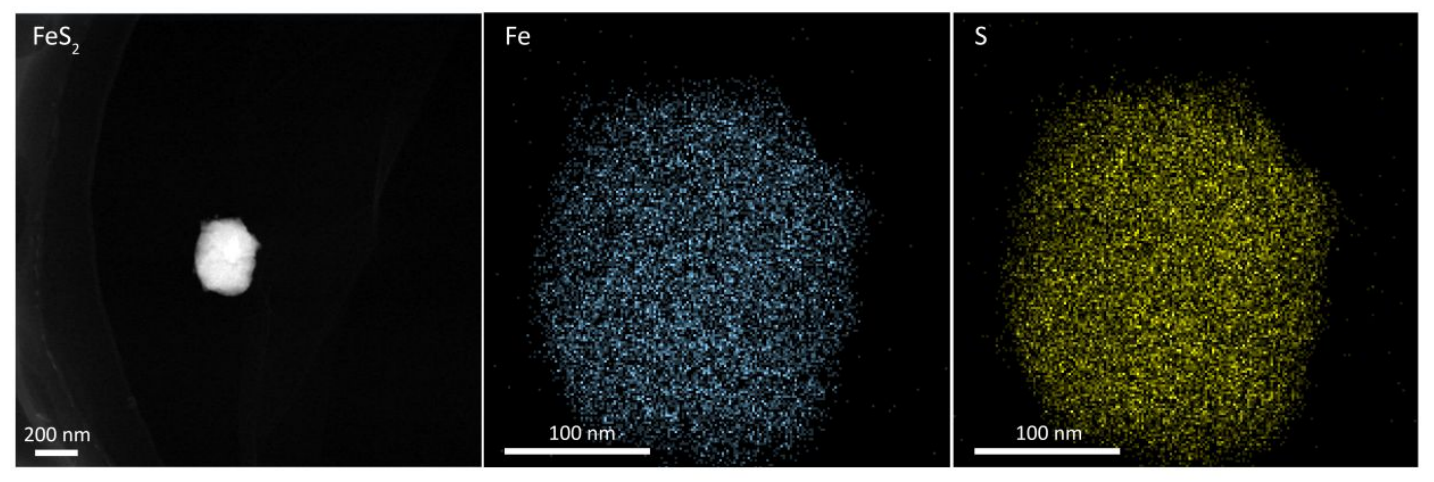

Figure S2. STEM images of $\mathrm{FeS}_{2}$. 


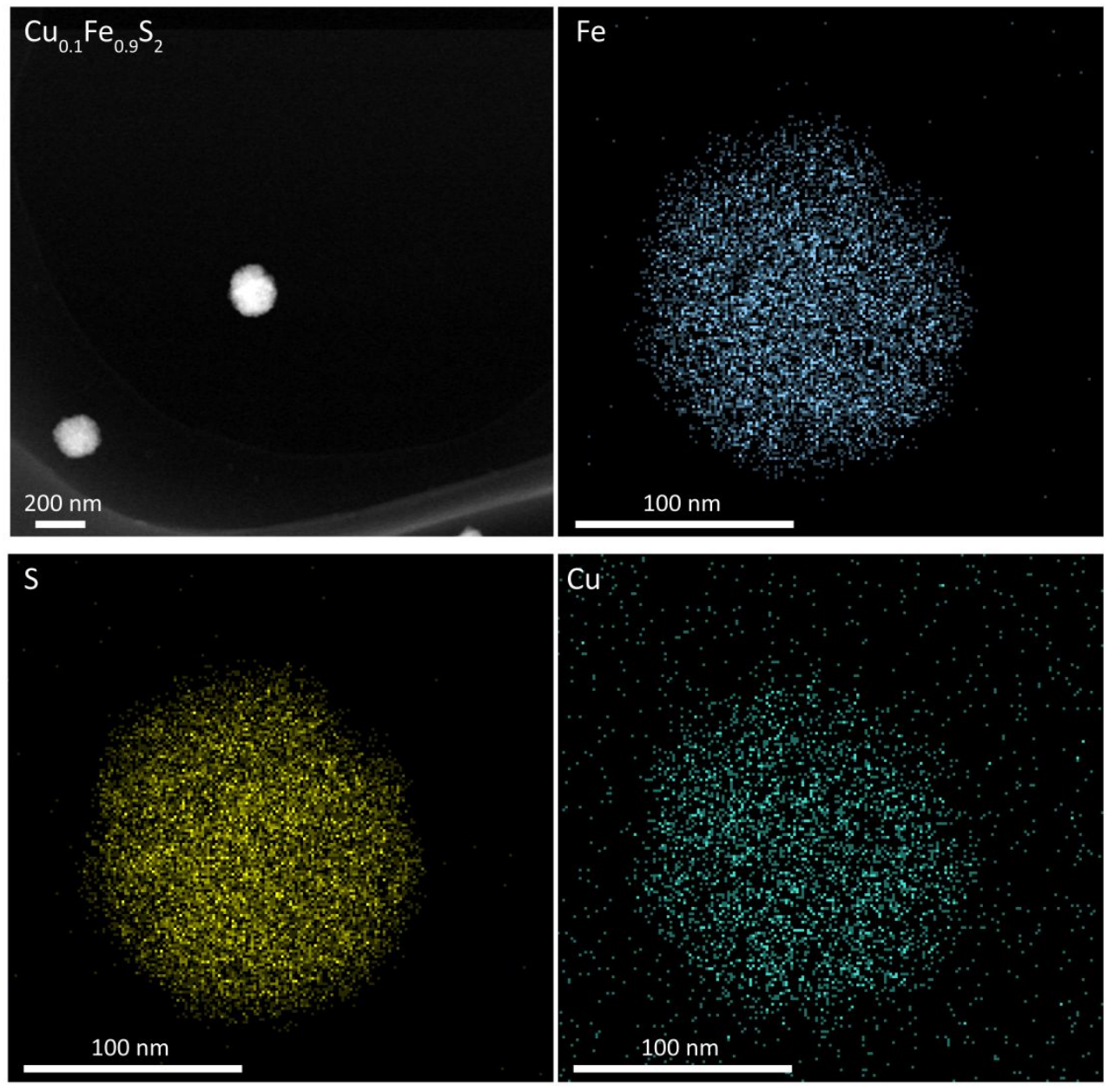

Figure S3. STEM images of $\mathrm{Cu}_{0.1} \mathrm{~F}_{\mathrm{e} 0.9} \mathrm{~S}_{2}$. 


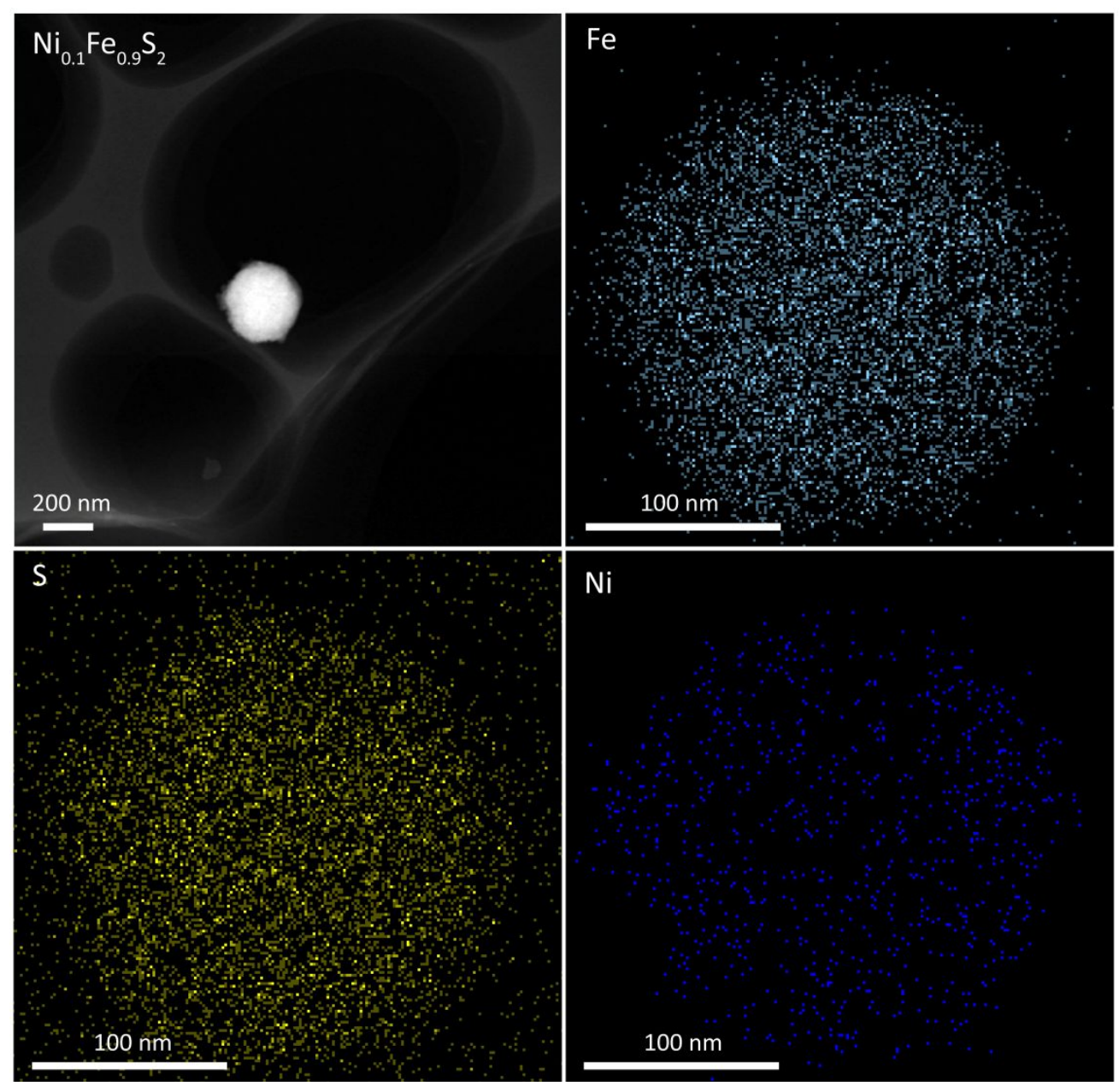

Figure S4. STEM images of $\mathrm{Ni}_{0.1} \mathrm{~F}_{\mathrm{e} 0.9} \mathrm{~S}_{2}$. 


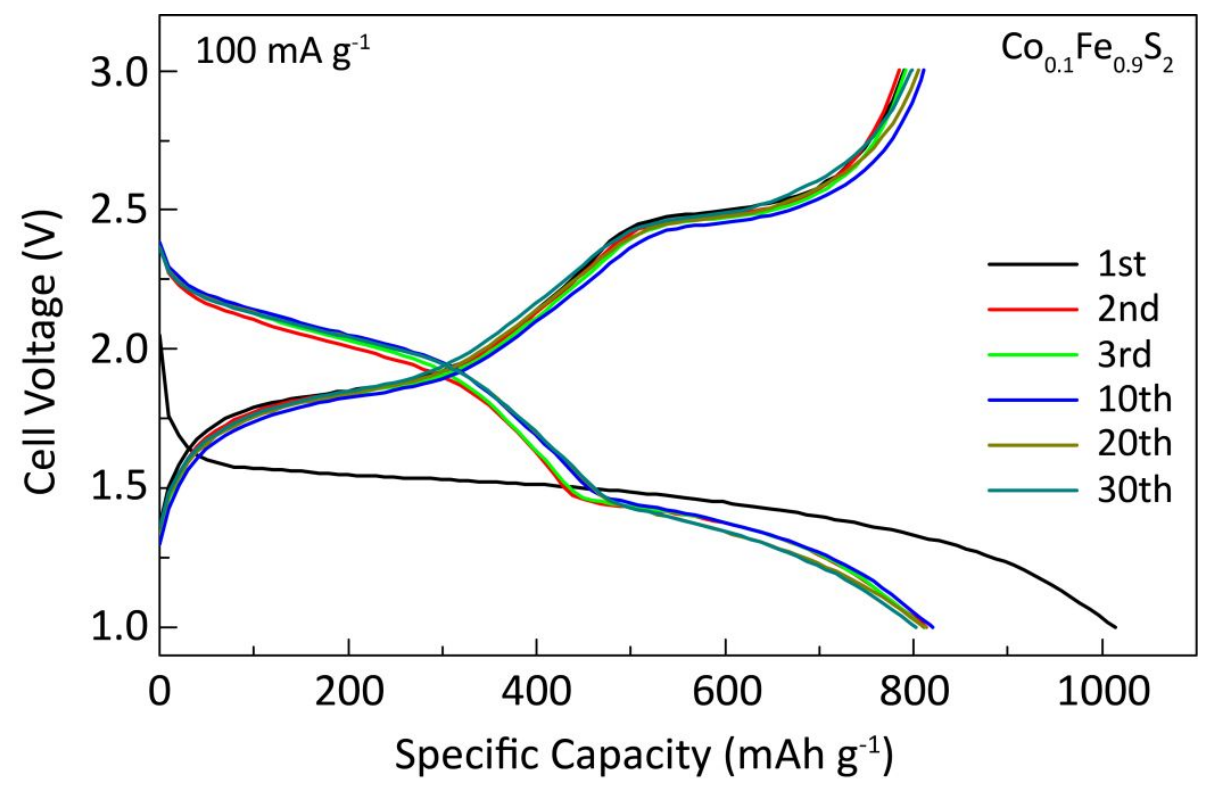

Figure S5. Galvanostatic discharge/charge profile of $\mathrm{Co}_{0.1} \mathrm{Fe}_{0.9} \mathrm{~S}_{2}$ electrode at current density of $100 \mathrm{~mA} \mathrm{~g}^{-1}$. 
Table S1. Comparison of electrochemical performance of $\mathrm{FeS}_{2}$ in all-solid-state lithium battery

\begin{tabular}{ccccc}
\hline Cathode & Temperature & Current density & Capacity $\left(\mathrm{mAh} \mathrm{g}^{-1}\right)$ & Reference \\
\hline $\mathrm{FeS}_{2}$ & $60^{\circ} \mathrm{C}$ & $0.1 \mathrm{C}$ & $\sim 900$ (20 cycles) & {$[3]$} \\
$\mathrm{FeS}_{2}$ & $30^{\circ} \mathrm{C}$ & $0.1 \mathrm{C}$ & $\sim 750$ (20 cycles) & {$[3]$} \\
$\mathrm{FeS}_{2}$ & $20^{\circ} \mathrm{C}$ & $0.09 \mathrm{C}$ & $\sim 800(20$ cycles $)$ & {$[18]$} \\
$\mathrm{FeS}_{2}$ & $60^{\circ} \mathrm{C}$ & $0.5 \mathrm{C}$ & $\sim 230(100$ cycles $)$ & {$[19]$} \\
$\mathrm{FeS}_{2} @ \mathrm{TiS}_{2}$ & $60^{\circ} \mathrm{C}$ & $0.5 \mathrm{C}$ & $\sim 440(20$ cycles $)$ & {$[19]$} \\
$\mathrm{Co}_{0.1} \mathrm{Fe}_{0.9} \mathrm{~S}_{2}$ & $\mathrm{RT}$ & $\sim 0.11 \mathrm{C}$ & $802.0(30$ cycles $)$ & \\
\cline { 3 - 5 } & & $\sim 0.55 \mathrm{C}$ & $625.9(20$ cycles $)$ & This work \\
\hline
\end{tabular}




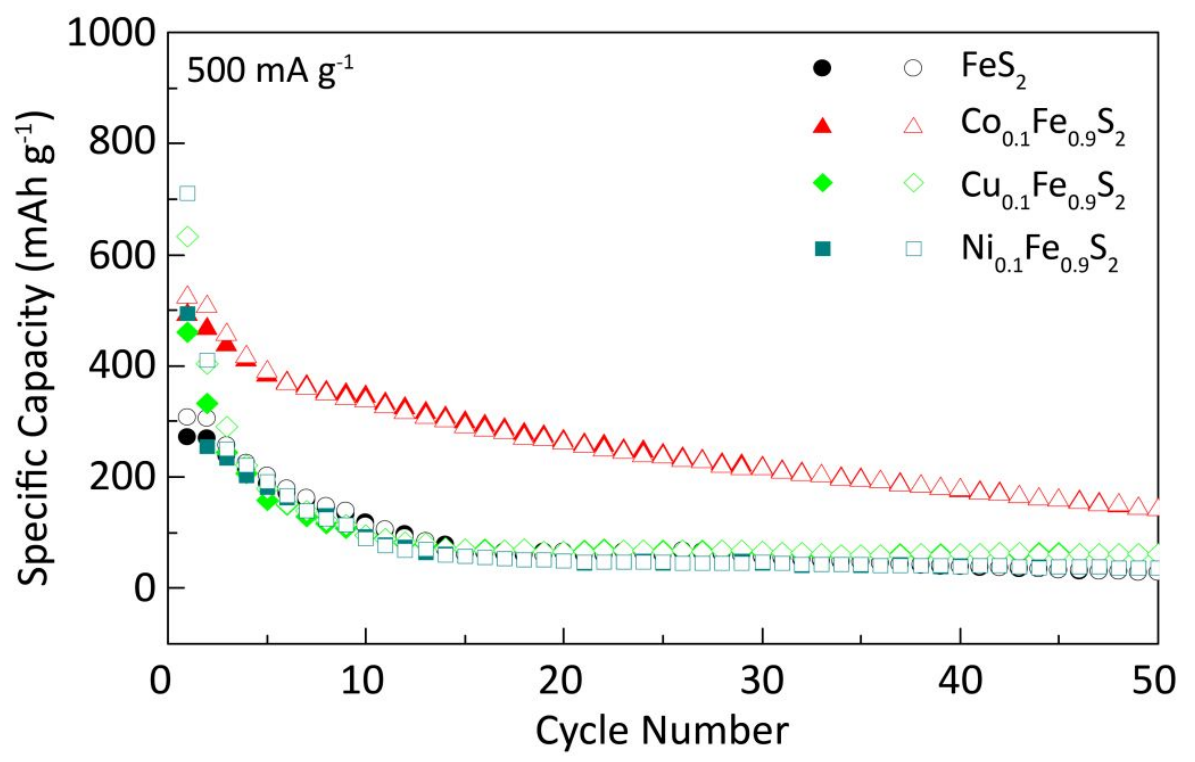

Figure S6. Cyclic performance of $\mathrm{FeS}_{2}$ and transition metal doped $\mathrm{FeS}_{2}$ at current density of 500 $\mathrm{mA} \mathrm{g}^{-1}$ in liquid cells. 


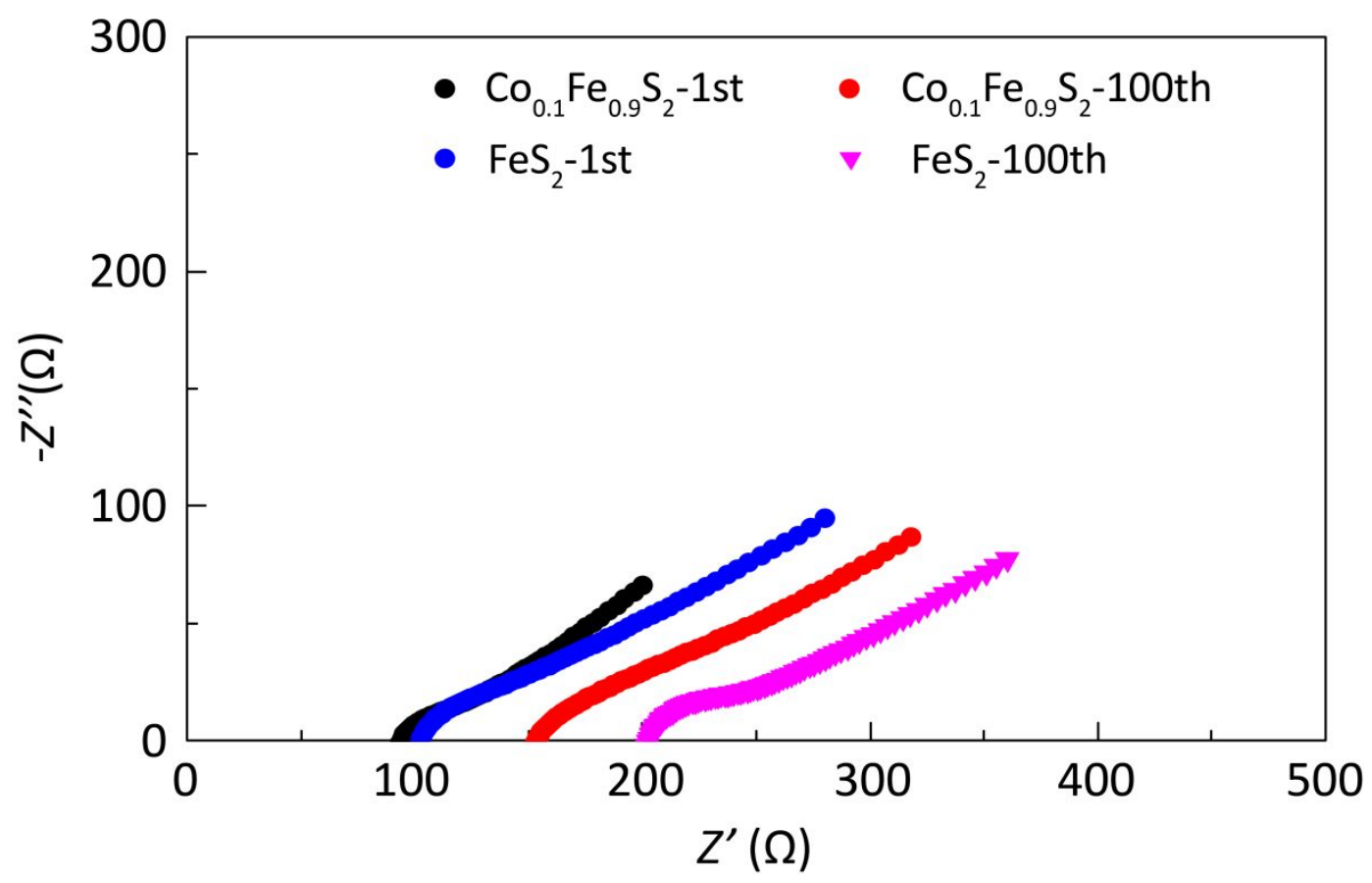

Figure S7. Nyquist plots for $\mathrm{FeS}_{2} / \mathrm{Li}_{10} \mathrm{GeP}_{2} \mathrm{~S}_{12}-75 \% \mathrm{Li}_{2} \mathrm{~S}-24 \% \mathrm{P}_{2} \mathrm{~S}_{5}-1 \% \mathrm{P}_{2} \mathrm{O}_{5} / \mathrm{Li}$ and $\mathrm{Co}_{0.1} \mathrm{Fe}_{0.9} \mathrm{~S}_{2} / \mathrm{Li}_{10} \mathrm{GeP}_{2} \mathrm{~S}_{12}-75 \% \mathrm{Li}_{2} \mathrm{~S}-24 \% \mathrm{P}_{2} \mathrm{~S}_{5}-1 \% \mathrm{P}_{2} \mathrm{O}_{5} / \mathrm{Li}$ battery after $1^{\text {st }}$ and $100^{\text {th }}$ cycle. 

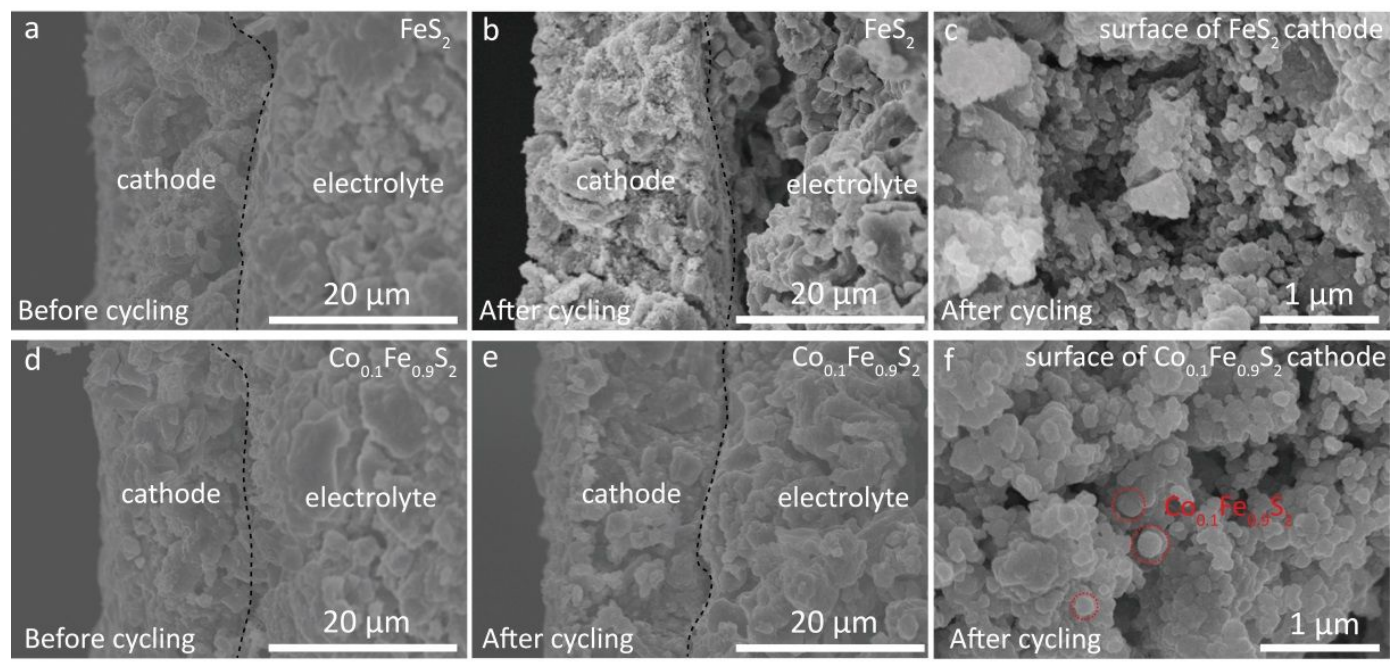

Figure S8. SEM images of cross-section of $\mathrm{FeS}_{2}$ cathode $/ \mathrm{Li}_{10} \mathrm{GeP}_{2} \mathrm{~S}_{12}$ (a) before cycling, (b) after 100th cycling and (c) surface of $\mathrm{FeS}_{2}$ cathode; SEM images of cross-section of $\mathrm{Co}_{0.1} \mathrm{Fe}_{0.9} \mathrm{~S}_{2}$ cathode $/ \mathrm{Li}_{10} \mathrm{GeP}_{2} \mathrm{~S}_{12}$ interface (d) before cycling, (e) after 100 cycles and (f) surface of $\mathrm{Co}_{0.1} \mathrm{Fe}_{0.9} \mathrm{~S}_{2}$ cathode. 